\section{Integrative Medicine \\ International}

Integr Med Int 2014;1:144-150

\section{DOI: $10.1159 / 000371467$}

Received: December 5, 2014

Accepted after revision: December 9, 2014

Published online: January 30, 2015

This is an Open Access article licensed under the terms of the Creative Commons AttributionNonCommercial 3.0 Unported license (CC BY-NC) (www.karger.com/OA-license), applicable to the online version of the article only. Distribution permitted for non-commercial purposes only.

\title{
Review
}

\section{Integrative Cognitive Reserve}

\author{
Wenfei Jiang Weidong Pan \\ Department of Neurology, Shuguang Hospital Affiliated to Shanghai University of \\ Traditional Chinese Medicine, Shanghai, China
}

\author{
Key Words \\ Cognitive reserve Brain reserve Contributing factors - Measurement of cognitive reserve \\ Multiple sclerosis · Integrative medicine
}

\begin{abstract}
The cognitive reserve (CR) against cognitive damage stems from a series of observations. The purpose of this study is to perform a systematic review to summarize the concept of CR. A variety of factors such as age, education level, reading ability, and vocabulary have an impact on the reserve according to a large amount of research and may influence CR. Furthermore, due to measurement difficulties, efforts to explore CR have been hindered. This is why different methods have been used to quantify reserve, such as multiple indicators multiple causes, residual variance in episodic memory performance, the Sole-Padulles Childhood Enrichment measure, the MiniMental State Examination, the Peabody Picture Vocabulary Test, the Hollingshead Two-Factor Index of Social Position, the Cattell Culture Fair Intelligence Test, and the Digit Span Subtests of the Wechsler Memory Scale. In addition, numerous studies explored the role of CR in various diseases, such as multiple sclerosis, hepatitis $C$, obesity, and sleep onset/maintenance difficulties. $C R$ plays a significant role in the morbidity of these diseases, and since integrative medicine may be able to modify $C R$, it might be an effective tool to prevent these serious diseases.
\end{abstract}

(C) 2015 S. Karger AG, Basel

\section{Introduction}

The concept of reserve against brain damage that originated from a series of observations indicates that the reserve does not appear to have a direct relationship between the degree of brain pathology or brain damage and the clinical manifestation of these damages $[1,2]$. Various concepts concerning neurological reserve, such as morbidity threshold, disease compensation, neuronal or brain reserve, and cognitive reserve (CR) have been proposed. CR parallels the concept of brain reserve in that it is a potential mechanism for coping with brain damage [2]. In the threshold model, the reserve capacity typically consists of additional synapses or an increased number of redundant neuronal networks [1]. CR focuses more on 
the 'software'. This could consist of the ability of the cognitive paradigm underlying a task to sustain disruption and still operate effectively. Alternately, this could consist of the ability to use alternate paradigms to approach a problem when the more standard approach is no longer in operation [1]. CR might be translated into differential susceptibility to factors that disrupt performance. The brain actively attempts to compensate for the challenge represented by the brain damage, which is the active model of CR. The active model of reserve may pay more attention to the mode in which tasks are processed as opposed to underlying physiologic differences [3]. It might take the form of using the brain network or a cognitive paradigm that is more efficient or flexible, and thus less susceptible to disruption. In essence, a person who uses a brain network more efficiently, or who is more capable of calling up an alternative brain network or cognitive strategies in sake of increased demand, may have more CR and might have a more effective performance over a longer period of time in the face of brain pathology [3]. In this paper, we review the studies on CR, and discuss the contributing factors, the clinical and pathological measurements of CR, and the contributions of CR for our body. Most of the research described is based on recent studies.

\section{Factors Contributing to CR}

Many factors might modulate CR. Sumowski and Leavitt [4] suggested that heritable (larger maximal lifetime brain growth, MLBG) and environmental (greater intellectual enrichment) factors attenuate the negative effect of disease burden on cognitive status. MLBG is typically measured by head size or intracranial volume (adjusted for sex). Persons who have a larger MLBG can lose more brain volume before reaching this critical threshold associated with cognitive impairment, suggesting that individuals with larger heads might have more CR than those with smaller heads. Intellectually enriching lifestyles are usually estimated with educational attainment or vocabulary. Vocabulary tests estimate semantic knowledge acquired through enriching activities such as education, occupation, and reading. That is, persons with larger MLBG, greater vocabulary knowledge, and/or greater early life participation in cognitive leisure activities (e.g. reading, hobbies) are better able to cope with multiple sclerosis (MS) without cognitive impairment. The impact of intellectual enrichment on cognitive status may stem from more efficient patterns of brain function. Mondini et al. [5] believe that CR could be defined as the accumulation of experiences, abilities, knowledge and changes that occur throughout the lifespan. One of the most difficult changes in life is the experience of emigrating to a foreign country. Emigration might act as an environmental factor that enriches individuals' lifestyle and reflects itself in the amount of their CR. Bilingualism may contribute to CR in normal aging. However, there is currently no neuroimaging evidence to suggest that lifelong bilingualism can help retain normal cognitive functioning in the face of age-related neurodegeneration. Gold et al. [6] explored this issue by comparing white matter (WM) integrity and gray matter (GM) volumetric patterns in older adult lifelong bilinguals $(n=20)$ and monolinguals $(n=20)$. The groups were matched on a range of relevant cognitive test scores and on the established CR variables of education, socioeconomic status, and intelligence. Participants underwent high-resolution structural imaging for assessment of GM volume and diffusion tensor imaging or magnetic resonance imaging (MRI) for assessment of WM integrity. The results indicated significantly lower microstructural integrity in the bilingual group in several WM tracts. In particular, compared to their monolingual peers, the bilingual group showed lower fractional anisotropy and/or higher radial diffusivity in the inferior longitudinal fasciculus/inferior fronto-occipital fasciculus bilaterally, the fornix, and multiple portions of the corpus callosum. There were no group differences in GM volume. The results suggest that lifelong bilingualism contributes to CR and protects against WM integrity declines in aging [6]. 


\section{Measurement of CR}

There are a variety of methods used to measure CR. Efforts to explore reserve have been hindered by measurement difficulties. Reed et al. [7] considered quantifying reserve as residual variance in episodic memory performance that remains after accounting for demographic factors and brain pathology (whole brain, hippocampal, and WM hyperintensity volumes). This residual variance represents the discrepancy between an individual's predicted and actual memory performance. Zahodne et al. [8] extended these methods to a larger, community-based sample and investigated whether the residual reserve variable is explained by age, predicts longitudinal changes in language as well as dementia conversion independent of age. The results support this operational measure of CR. The residual reserve variable was associated with higher reading ability, lower likelihood of meeting criteria for mild cognitive impairment, lower odds of dementia conversion independent of age, and less decline in language abilities over 3 years. The residual reserve variable moderated the negative impact of memory variance explained by brain pathology on language decline. One of the methods is using the Sole-Padulles Childhood Enrichment measure of passive CR, which includes the respondent's education level, the education levels of the parents, and childhood participation in music, arts, and social activities. Active CR was measured by the Stern Leisure Activities measure, which assesses current hobbies, and cultural as well as leisure activities. These measurements were of reliability and validity [9]. Mondini et al. [5] qualified CR through the Italian version of the Cognitive Reserve Index questionnaire (CRIq). This questionnaire collects information on three different aspects of an individual CRI score and three different subscores for each section (CRI-Education, CRI-Working Activity, and CRI-Leisure Time). It includes these three aspects of life as they are the most frequently used proxies according to the literature. CRI-Education refers to years of schooling plus possible training courses (each lasting at least 6 months); the raw score of this section is the sum of these two values. CRIWorking Activity registers the professions carried out during adulthood, classified into five different levels of working activities according to the degree of intellectual involvement and personal responsibility: unskilled, manual work (e.g., farmer, car driver, call center operator); skilled manual work (e.g., craftsman, clerk, hairdresser); skilled nonmanual or technical work (e.g., trader, kindergarten teacher, real estate agent); professional occupation (e.g., lawyer, psychologist, physician), and highly intellectual occupation (e.g., university professor, judge, top manager). The raw score of this section was the result of the years of working activity multiplied by the cognitive level of the occupation. CRI-Leisure Time: cognitively stimulating occupations carried out during leisure time (outside work or school time). The CRI showed a satisfactory correlation with the three subscores, CRI-Education, CRI-Working Activity and CRI-Leisure Time: $r=0.77, r=0.78$, and $r=0.72$, respectively. The correlation between the subscore was not high: CRI-Education and CRI-Working Activity: $r=0.44$; CRI-Education and CRI-Leisure Time: $r=0.30$, and CRI-Working Activity and CRI-Leisure Time: $r=0.32$. The first was significantly higher than the other two $(\mathrm{z}=2.78, \mathrm{p}<0.01$ and $\mathrm{z}=2.40, \mathrm{p}<0.01$, respectively). As expected, the correlation between CRI and CRI-Education was significantly lower for the elderly with respect to the young and adults. The correlation between CRI and CRIWorking Activity was significantly low. Adults also showed a significantly low correlation between CRI and CRI-Leisure Time. CRIq is an efficient and reliable tool for measuring CR. In particular, it is short and easy to administer, and thus can easily be included in standard assessments without too much effort for the subject in terms of both time and cognitive resources. It also overcomes the major limitations found in studies estimating CR. The CRIq takes into account not only the number of years during which the activities were carried out, but also their frequency. Unlike other instruments, which only measure activities late in life, the CRIq can be administered at any age. Lastly, since the measurement of CR is not one of 
performance, a significant and interesting feature of CRIq is that it can also be administered to relatives or close friends, if an individual cannot be interviewed for any reason (e.g., brain impairment, aphasia, coma, dementia, etc.). Above all, the CRIq provides a standardized and psychometrically controlled measure of $\mathrm{CR}$, allowing its extensive employment in both experimental research and clinical practice. In fundamental research, the CRIq represents a single index to compare data and results from different studies. All investigations evaluating cognitive abilities could benefit from it in place of education only, in order to assess individual performance better. The CRIq may also be used to validate new psychometric tests (only age and education are usually considered). In clinical settings, the CRIq could be used to diagnose senile dementia, comparing CRI and cognitive performance. In subjects with high CRI scores, the richness of neuronal synaptic tissue and its plasticity acts as a reserve, compensating for the atrophy of GM and covering clinical pathological signs. Thus, poor performance on cognitive tests, but still within the normal range, must be considered suspect in cases with a high CRI. Conversely, poor performance combined with a low CRI is expected. The CRIq could also enable psychologists to gain a more complete image of their patients and their lifestyles, including information from standard assessments that usually depend on the clinician's experience [10].

Giogkaraki et al. [11] tested the CR hypothesis by quantifying CR. A neuropsychological battery was administered to 383 Greek-Cypriot older adults. A multiple indicators multiple causes (MIMIC) latent construct was utilized to define CR incorporating three indicators: years of education, vocabulary, and reading performance. Findings from two structural equation models supported the moderating role of CR in reducing the direct negative effect of age on verbal episodic memory and on executive function. Zihl et al. [12] observed CR as individual improvement in cognitive performance (gain) in a complex testing-the-limits paradigm, the digit symbol substitution test (DSST) with 10 repeat measurements, in 140 younger (20-30 years) and 140 older (57 years or older) subjects. They also assessed attention, memory and executive function as well as mood and personality traits as potential factors that influence CR. They found that both younger and older subjects showed significant gains, which were significantly correlated with the speed of information processing, verbal short-term memory, and visual problem solving in the older group only. Gender, personality traits, and mood did not significantly influence gains in either group. Surprisingly, about half of the older subjects performed at the level of the younger group, suggesting that interindividual differences in CR are possibly age independent. They propose that these findings may also be understood as an indication that one-time standard individual measurements do not allow assessment of CR, and that the use of DSST in a testing-thelimits paradigm is a valuable assessment method for CR in young and elderly subjects. Galioto et al. [13] used an estimate of premorbid intelligence as a measure of CR. Premorbid intelligence has been well documented as a robust measure of CR. To assess premorbid intelligence, a computerized adaptation of the Spot the Word Test was used. In this task, participants are presented with two words, one valid English word and a nonword foil. The total correct score is entered into a regression formula including education and age to estimate the intelligence quotient. This estimate is strongly correlated with the Wechsler Adult Intelligence Scale and served as the measure of CR. Gold et al. [6] used the following scales to test CR: (1) The Mini-Mental State Examination (MMSE), which is a 30-point test used to screen for cognitive impairment. It assesses orientation to time and place as well as basic memory functions. (2) The Peabody Picture Vocabulary Test (PPVT-III), which is a culture-fair measure used to assess proficiency in English. There are a total of 204 test items. Test items are presented on pages containing four black-and-white pictures. Participants are read a word and asked to choose the picture on that page that best corresponds to the word. (3) The Hollingshead Two-Factor Index of Social Position (ISP), which was 
used as a measure of socioeconomic status. The ISP is based on an individual's occupation and highest level of formal education. It is calculated by assigning numeric values from 1 to 7 to an individual's occupation and education. Scores are then weighted by multiplying by 7 (occupation) and 4 (education). Values are summed to produce a social index. Lower values represent a higher earning occupation and more years of education. (4) The Cattell Culture Fair (CCF) Intelligence Test, which is a test of fluid intelligence that is not influenced by cultural background or verbal ability. The CCF consists of 50 items and assesses inductive reasoning about relationships in shapes and figures. (5) The Digit Span Subtests of the Wechsler Memory Scale assess verbal memory. Participants are read digit lists aloud and are then instructed to repeat each set of digits verbally in the same order (digit forward) and in reverse order (digit backward). In both situations, the test is performed twice. Standard termination procedures were followed and the totals for the digit forward and digit backward sets were based on the number of trials that were accurately reported in the correct order.

A more objective measurement is also available. Querbes et al. [14] used the Normalized Thickness Index (NTI), which measures cortical thickness, on a large sample of patients from the Alzheimer's Disease Neuroimaging Initiative (ADNI) database and found evidence that structural MRI can be used to detect subtle structural changes that help predict the subject's outcome up to 24 months before clinical diagnosis. They also investigated the CR hypothesis on their population and showed that subjects with a higher education level had a significantly thinner cortex than less educated subjects with the same level of cognitive performance. The NTI also correctly predicted the evolution to Alzheimer's disease for $76 \%$ of amnestic mild cognitive impairment subjects after a cross-validation, thus showing an advantage over cognitive scores (range, 63-72\%).

\section{Effects of CR in Diseases}

\section{Impact of CR in $M S$}

Cognitive impairment is common among persons with MS, but some patients are able to withstand considerable disease burden (e.g. WM lesions, cerebral atrophy) without cognitive impairment. A larger MLBG and intellectual enrichment attenuate the negative relationship between MRI estimates of disease burden and cognitive status. Furthermore, the correlation between cognitive status and MS disease burden is stronger in samples with lower MLBG and/or lower intellectual enrichment. Future clinical research aimed at identifying the best MRI predictors of cognitive status must consider the moderating effect of reserve on this relationship, especially when comparing results across study samples with differing levels of reserve (estimated with MLBG or intellectual enrichment) [4]. Schwartz et al. [9] applied two health outcome measures, the Symptom Inventory and the Performance Scales, collected biannually over 1 and 6 years, respectively, to examine the relationship between CR and MS. Active and passive CRs were measured using the Stern Leisure Activities and Sole-Padulles Childhood Enrichment tools, respectively. Linear regression, $\chi^{2}$, multilevel random effects modeling, and classification as well as regression tree modeling were used to compare cross-sectional means, disease course by CR, longitudinal trajectories, and active CR item endorsement by disability groups, respectively. They found that patients with a high active reserve had a lower symptom burden than those with a low active reserve independent of passive reserve. CR was associated with the course of disease, such that high active patients were overrepresented among relapsing-remitting patients, and underrepresented among patients with progressive disease. Longitudinal modeling revealed a significant interaction of active reserve and time in mobility, fatigue, and overall 
disability in the whole sample. Among patients whose disability trajectories changed over time, active CR was associated with less deterioration and passive CR had no apparent effect in the longitudinal analyses. Active CR scores across disability groups had a similar range but comprised different items, indicating that patients maintain active CR with different activities as the disease progresses.

Protective Effects of Higher CR for Individuals Infected with Hepatitis $C$

Higher levels of CR can be protective against the neuropsychological manifestation of neural injury across a variety of clinical disorders. Sakamoto et al. [15] studied the protective effects of higher CR in individuals infected with hepatitis C. Thirty-nine HCV-infected participants were classified as having either high $(n=19)$ or low $(n=20)$ CR based on educational attainment, oral word reading, and IQ scores. A sample of 40 demographically comparable healthy adults was also included. All participants completed the Neuropsychological Assessment Battery, Delis-Kaplan Executive Function System, and Behavioral Rating Inventory of Executive Function, Adult Version (BRIEF-A). Linear regression analyses controlling for gender, depression, and lifetime substance abuse disorders found significant effects in the HCV/CR group in verbal fluency, executive functions, and daily functioning $\mathrm{T}$ scores, but not in learning or the BRIEF-A. Meanwhile, comparison of the groups revealed that the HCV group with low CR performed significantly below the HCV group with high CR and healthy adult cohorts, who did not differ from one another. These findings indicate that higher levels of CR may be a protective factor in the neurocognitive and real-world manifestation of neural injury commonly associated with HCV infection.

\section{CR in Obese Individuals}

Obesity is an established risk factor for cognitive impairment. Theories of CR suggest that premorbid factors, such as intellectual ability, may attenuate the expression of cognitive impairment due to age or disease. Galioto et al. [13] examined whether CR, defined as estimated premorbid intellectual ability, moderates the relationship between obesity and cognitive function in obese adults. Participants without major medical or psychological conditions completed a computerized battery of neuropsychological tests. Hierarchical regression models found a significant interaction between BMI and CR for attention/executive function and memory, suggesting that CR attenuates the expression of obesity-related cognitive impairment.

\section{CR and Sleep Onset/Maintenance Difficulties}

This study examined the relationship between cognitive function and sleep onset/maintenance difficulties (SO/MD) in nondemented older adults. Zimmerman et al. [16] hypothesized that SO/MD negatively impacts cognition and that older adults with lower education would be especially vulnerable to its effects. Their sample consisted of 549 older adults from the Einstein Aging Study (EAS), a community-based cohort. Participants underwent a neuropsychological assessment and completed a sleep questionnaire. SO/MD (present or absent) and education (lower: $<12$ years; higher: $\geq 12$ years) were identified as between-subject factors, and age, ethnicity, gender, depression, as well as cardiovascular comorbidities were found to be covariates. The average age of the participants was 79.76 years (range, 57-97). Fifty-seven percent of the sample met the criteria for SO/MD. Among the participants with SO/MD, those with lower education performed more poorly on a test of category fluency than participants with higher education. Among older adults without SO/MD, educational attainment had no measurable effect on cognition. Consistent with the CR hypothesis, older adults with a lower education level appeared to be selectively vulnerable to the negative effects of SO/MD on tests of verbal fluency. 


\section{Conclusions}

The concept of CR, one important reserve in the human body, contributes very interesting and useful ideas and concepts to the understanding of the human brain. It is a very important factor to prevent the brain from suffering from cognitive impairment, and to improve the state of serious diseases such as MS and obesity. If the pathogenic factors of CR can be controlled, the appearance of cognitive impairment or dementia can perhaps be delayed or stopped. Integrative medicine [2], a powerful and integrative technique that is used to treat many diseases, especially in East Asia, may have the ability to increase CR. Future studies should test whether CR can be altered by integrative medicine in long-term studies in integrative areas.

\section{Acknowledgments}

This study was sponsored and supported by the National Natural Science Foundation of China (81373619).

\section{Disclosure Statement}

The authors declare that there are no conflicts of interest regarding the publication of this article.

\section{References}

1 Stern Y: What is cognitive reserve? Theory and research application of the reserve concept. J Int Neuropsychol Soc 2002;8:448-460.

2 Pan W: Integrative reserve and integrative medicine. Integr Med Int 2014;1:119-121.

3 Stern Y: The concept of cognitive reserve: a catalyst for research. J Clin Exp Neuropsychol 2003;25:589-593.

4 Sumowski JF, Leavitt VM: Cognitive reserve in multiple sclerosis. Mult Scler 2013;19:1122-1127.

5 Mondini S, Guarino R, Jarema G, et al: Cognitive reserve in a cross-cultural population: the case of Italian emigrants in Montreal. Aging Clin Exp Res 2014;26:655-659.

6 Gold BT, Johnson NF, Powell DK: Lifelong bilingualism contributes to cognitive reserve against white matter integrity declines in aging. Neuropsychologia 2013;51:2841-2846.

7 Reed BR, Mungas D, Farias ST, et al: Measuring cognitive reserve based on the decomposition of episodic memory variance. Brain 2010;133:2196-2209.

-8 Zahodne LB, Manly JJ, Brickman AM, Siedlecki KL, Decarli C, Stern Y: Quantifying cognitive reserve in older adults by decomposing episodic memory variance: replication and extension. J Int Neuropsychol Soc 2013;19:854-862.

-9 Schwartz CE, Quaranto BR, Healy BC, Benedict RH, Vollmer TL: Cognitive reserve and symptom experience in multiple sclerosis: a buffer to disability progression over time? Arch Phys Med Rehabil 2013;94:1971-1981.

10 Nucci M, Mapelli D, Mondini S: Cognitive Reserve Index questionnaire (CRIq): a new instrument for measuring cognitive reserve. Aging Clin Exp Res 2012;24:218-226.

11 Giogkaraki E, Michaelides MP, Constantinidou F: The role of cognitive reserve in cognitive aging: results from the neurocognitive study on aging. J Clin Exp Neuropsychol 2013;35:1024-1035.

12 Zihl J, Fink T, Pargent F, Ziegler M, Buhner M: Cognitive reserve in young and old healthy subjects: differences and similarities in a testing-the-limits paradigm with DSST. PLoS One 2014;9:e84590.

-13 Galioto RM, Alosco ML, Spitznagel MB, Stanek KM, Gunstad J: Cognitive reserve preserves cognitive function in obese individuals. Neuropsychol Dev Cogn B Aging Neuropsychol Cogn 2013;20:684-699.

14 Querbes O, Aubry F, Pariente J, et al: Early diagnosis of Alzheimer's disease using cortical thickness: impact of cognitive reserve. Brain 2009;132:2036-2047.

15 Sakamoto M, Woods SP, Kolessar M, et al: Protective effects of higher cognitive reserve for neuropsychological and daily functioning among individuals infected with hepatitis C. J Neurovirol 2013;19:442-451.

-16 Zimmerman ME, Bigal ME, Katz MJ, Brickman AM, Lipton RB: Sleep onset/maintenance difficulties and cognitive function in nondemented older adults: the role of cognitive reserve. J Int Neuropsychol Soc 2012;18:461-470. 